\title{
La formación docente en el estado de Chihuahua: Entre el desafío sanitario y la resiliencia docente
}

\author{
Teacher training in the state of Chihuahua: Between \\ the health challenge and teacher resilience
}

Evangelina Cervantes Holguín ${ }^{1}$, Pavel Roel Gutiérrez Sandoval ${ }^{2}$ y Cely Celene Ronquillo Chávez ${ }^{3}$

Fecha de recepción: 9 de marzo de 2021

Fecha de aceptación: 13 de mayo de 2021

1 - Nacionalidad: Mexicana. Grado: Doctorado en Educación. Adscripción: Universidad Autónoma de Ciudad Juárez. (D) ORCID: https://orcid.org/0000-0001-6980-2210 | Correo electrónico: linacervantes@hotmail.com

2 - Nacionalidad: Mexicana. Grado: Doctorado en Educación. Adscripción: Universidad Autónoma de Ciudad Juárez. (D) ORCID: https://orcid.org/0000-0003-0437-1549 | Correo electrónico: pavel.gutierrez@uacj.mx

3 - Nacionalidad: Mexicana. Grado: Doctorado en Educación. Adscripción: Universidad Autónoma de Ciudad Juárez. (D) ORCID: https://orcid.org/0000-0002-7902-4544 | Correo electrónico: cronquil@uacj.mx 


\section{Resumen}

El artículo propone recuperar la respuesta de las Instituciones Formadoras y Actualizadoras de Docentes en el estado de Chihuahua ante los diversos desafíos impuestos por la pandemia del coronavirus (COVID-19). El ejercicio cualitativo analiza la experiencia de 10 instituciones a partir de la voz de sus estudiantes, docentes $y$ directivos en torno a los cambios en los procesos académicos, administrativos y organizativos. Se concluye que la pandemia ha afectado de diferente manera y con distinta intensidad a cada institución. Pese a los logros, la experiencia analizada devela el relativo éxito del uso de las plataformas virtuales ante tres condiciones básicas: conectividad, competencias tecnológicas y habilidades socioemocionales del profesorado. Destaca la importancia de implementar acciones tutoriales, de resiliencia o de concienciación de las necesidades, sentimientos y malestares de docentes y estudiantes. Resulta oportuno recuperar las experiencias de otras instituciones e interpelar especialmente a estudiantes, tesistas y egresados.

Palabras clave: Acceso a la educación, aprendizaje en línea, educación a distancia, formación de docentes, tecnología educacional.

\section{Abstract}

The article proposes to recover the response of the Teacher Training and Updating Institutions in the state of Chihuahua regarding the various challenges imposed by the Coronavirus Disease (COVID-19). The qualitative exercise analyzes the experience of 10 institutions based on the voice of their students, teachers, and principals regarding changes in academic, administrative, and organizational processes. It is concluded that the pandemic has affected each institution in different ways and with diverse intensity. Despite the achievements, the experience analyzed reveals the relative success of using virtual platforms in the face of three basic conditions: connectivity, technological competencies, and socio-emotional skills of the teaching staff. It highlights the importance of implementing tutoring, resilience, or awareness actions of teachers and students' needs, feelings, and sufferings. It is opportune to recover the experiences of other institutions and to question especially students, thesis students, and graduates.

Keywords: Access to education, online learning, distance education, teacher education, educational technology. 


\section{Introducción}

$\mathrm{E}$ n marzo del 2020, el gobierno mexicano decretó suspender las clases presenciales. Ante el desafío sanitario provocado por la Covid-19, las escuelas quedaron vacías y el profesorado inició un agotador recorrido que, agravado por la ausencia de espacios para la clase y las dificultades de acceso, transmisión u organización de tiempos y espacios, se reveló como un difícil momento para todos (Alves et al., 2020). De la noche a la mañana, las escuelas se convirtieron "en centros escolares de educación a distancia" (Murillo y Duk, 2020, p. 13).

En este mundo convulso e incierto donde las actividades cotidianas han sido reemplazadas por encuentros remotos, es pertinente reflexionar sobre los nuevos retos para la formación docente y sus instituciones. El presente trabajo tiene como propósito recuperar la respuesta de las Instituciones Formadoras y Actualizadoras de Docentes (IFAD) a los diversos desafíos impuestos por la contingencia sanitaria en el estado de Chihuahua. El ejercicio pretende sistematizar críticamente las acciones implementadas para la continuidad académica, a partir de cuatro supuestos: 1. La excepcionalidad educativa consecuencia del SARS-CoV-2 modificó la formación docente; 2. En respuesta, las IFAD ajustaron sus procesos académicos, administrativos y organizacionales; 3 . La experiencia devela la presencia de necesidades históricas y actuales que precisan atención, y; 4. La pandemia, más que representar un periodo de crisis, puede sentar las bases para una acción formativa inédita en la región.

En el país, la formación docente, "ha tenido una profunda historia en la cual se han desarrollado diversos programas... para favorecer el acceso de los docentes a una oferta pertinente para su quehacer educativo" (Cordero et al., 2017, p. 31). Esta historia se ha configurado en medio de situaciones sociales, económicas, políticas y culturales diversas que, ahora, se incrementan en medio de la pandemia. Esta emergencia sanitaria, resultado de las políticas y estilos de vida acordes al neoliberalismo, no solo ha representado una alternativa para la continuidad académica, sino una oportunidad de bonanza para las empresas de la economía digital en los rubros de acceso a herramientas digitales y la capacitación para su uso (CAF, 2020; Díaz-Barriga y Barrón-Tirado, 2020).

En términos generales, la formación docente "no sólo es un asunto central para mejorar la educación, sino constituye un mecanismo fundamental para renovar el sistema educativo al posibilitar espacios para que el profesorado analice y transforme sus prácticas" (Cervantes y Sandoval, 2011, pp. 2-3). A nivel nacional y estatal, ésta se despliega en una diversidad de modalidades, según se trate de formación inicial, actualización, capacitación y superación profesional, que se desarrollan con la colaboración de distintas instituciones que, en realidades heterogéneas, hacen frente a los desafíos de la educación a distancia.

\section{Referentes teóricos}

En México, los servicios de formación docente se agrupan en cuatro modalidades, sobre la idea de que el desarrollo profesional docente alude a un proceso inacabado de aprendizaje y crecimiento continuo que se da a lo largo de la vida, comenzando en la formación inicial y seguida de oportunidades de actualización, capacitación y superación profesional (Instituto Nacional para la Evaluación de la 
Educación (INEE), 2018a). Así, cada periodo formativo cumple con funciones y propósitos específicos a fin de conformar una oferta diversa, suficiente, relevante, pertinente, de calidad, con apego a los principios de equidad, inclusión y gratuidad, así como en favor de la atención a los temas prioritarios y de relevancia social (Santibáñez et al., 2018).

A partir del 2017, la Secretaría de Educación Pública (SEP) amplió la noción inicial de las IFAD para incorporar a otras instancias encargadas de esta tarea. Así, se propuso el término de Instituciones Especializadas en Formación Pedagógica de Profesionales de la Educación (IEFP) que incluye a "las Escuelas Normales, Universidades Pedagógicas, Centros de Investigación Educativa, Asociaciones de Profesionales y Organismos nacionales o internacionales dedicados a la formación profesional, inicial y continua del personal educativo" (Cordero et al., 2017, p. 62).

En la trayectoria profesional, la formación inicial representa el punto de acceso a la docencia, por lo que:

debe orientarse a desarrollar en los futuros docentes habilidades para la reflexión continua, el trabajo colaborativo y la formulación de proyectos que vinculen su aprendizaje con la mejora de la escuela, aspectos que les permitirán seguir formándose y fortaleciendo su desempeño durante el servicio. (INEE, 2018a, p. 18)

En la actualidad, este tipo de formación se ofrece en "las escuelas normales, las universidades y las instituciones de educación superior" (SEP, 2017, p. 208). En el estado de Chihuahua, se reconocen como instituciones para la formación inicial: el Centro de Actualización del Magisterio, la Escuela Normal Experimental Miguel Hidalgo, la Escuela Normal Rural Ricardo Flores Magón, la Escuela Normal Superior Profr. José E. Medrano R. (ENSECH), la Escuela Normal Yermo y Parres, la Institución Benemérita y Centenaria Escuela Normal del Estado de Chihuahua Profr. Luis Urías Belderráin (IByCENECH) y, la Universidad Pedagógica Nacional del Estado de Chihuahua (UPNECH). Conjuntamente ofrecen a las y los jóvenes, con orientación a la docencia, una amplia oferta para formarse como profesionales de la educación especializados en educación inicial, preescolar, primaria, secundaria, indígena y especial.

La formación continua o en servicio — desarrollada en el país desde 1944 (Cordero et al., 2017)—, "se ofrece a todos los actores educativos a lo largo de su vida profesional, desde el ingreso hasta la jubilación" (p. 9), ya sea con la intención de actualizar, capacitar o especializar al profesorado mediante cursos, talleres, diplomados, proyectos docentes al interior de la escuela y los programas de posgrado.

La actualización "refiere a procesos de desarrollo, profundización y/o ampliación de la formación adquirida en la etapa de formación inicial, incorporando nuevos elementos (teóricos, metodológicos, instrumentales y disciplinares)" (SEP, 2008, p. 45). En Chihuahua, las instituciones encargadas de esta modalidad son el Centro de Actualización del Magisterio de Chihuahua (CAM), la ENSECH y la UPNECH. Por su parte, la capacitación alude a "los procesos a través de los cuales los profesionales de la educación se forman para atender de manera eficiente las innovaciones del sistema educativo, desde las distintas funciones que desempeñen, sean éstas curriculares, de gestión o tecnológicas" (SEP, 2008 , p. 45). Finalmente, la superación profesional apunta a "la formación destinada a quienes desean especializarse en campos diversos relativos al quehacer educativo y alcanzar mayores niveles de habilitación profesional y desarrollo dentro del SEM a través de estudios de especialización, maestría y 
doctorado" (SEP, 2008, p. 45). En este ámbito se encuentran el Centro Chihuahuense de Estudios de Posgrado (CCHEP), el Centro de Investigación y Docencia (CID), la ENSECH y la UPNECH.

Desde la segunda mitad del siglo XIX, en el estado de Chihuahua se han implementado diferentes proyectos para la formación docente que introducen visiones acerca del tipo de maestras/os que la sociedad requiere para la formación de los nuevos ciudadanos (Trujillo et al., 2018). En los albores del siglo XXI, Cervantes y Sandoval (2011) encontraron que, las IFAD se enfrentaban a diferentes desafíos y sugerían para su atención:

redimensionar la oferta educativa con base en diagnósticos que permitan conocer los requerimientos y necesidades de profesores, revisar los perfiles de egreso a fin de formar profesionales de la educación competentes para enfrentar con éxito la diversidad cultural y geográfica, integrar en los referentes teóricos el enfoque andragógico y la perspectiva de género, analizar la conformación redes y comunidades de aprendizaje, valorar las experiencias de microenseñanza, así como la figura del tutor en la formación para la investigación. (p. 8)

En México, la formación docente se caracteriza por presentar una serie de debilidades, como: insuficiente articulación de la formación inicial y continua, distancia entre los contenidos formativos y las necesidades del profesorado en sus diversos contextos, fragilidad de los conocimientos disciplinarios y las habilidades didácticas, limitada participación de las y los docentes en la definición de sus propios itinerarios formativos, entre otros (INEE, 2018a).

Para Navarro (2020), la pandemia vino a recrudecer las dificultades en que han funcionado las IFAD en la entidad: dependencia administrativa, legal, económica e incluso académica en el marco de una legislación formulada hace más de cuatro décadas. Los problemas de los últimos 30 años, más las circunstancias atípicas de la emergencia sanitaria, se agravan ante "la drástica disminución de presupuestos, tanto para las Instituciones Formadoras de Docentes, como para la capacitación y la formación continua, cuyos niveles marcan límites mínimos históricos" (párr. 2).

De acuerdo con la autoevaluación de las competencias docentes dirigida por Chávez et al. (2017), el profesorado de la Escuela Normal Rural Ricardo Flores Magón se valora solvente en la planificación de los procesos de enseñanza y aprendizaje, la selección de contenidos disciplinares, el diseño de procesos de evaluación, la práctica docente, la interacción pedagógica, la tutoría y el acompañamiento; sin embargo, reconoce debilidad en el dominio de una segunda lengua, el uso de las tecnologías de la información y el desarrollo de proyectos de investigación educativa. Al respecto, Jurado et al. (2017) descubrieron debilidades en las competencias digitales y la falta de acceso a infraestructura tecnológica entre las y los docentes de dos escuelas normales ubicadas en el estado. Por su parte, Cruz y Delgado (2019), profundizaron en las competencias investigativas del profesorado, tomando como referencia a la IByCENECH, encontrando que, pese a que las y los docentes reconocen en la investigación una herramienta para la mejora del desempeño académico, su participación en cuerpos académicos o redes de investigación es mínima.

Con un interés prospectivo para las IFAD en el estado de Chihuahua, Núñez et al. (2017) proponen avanzar en la construcción de una docencia pertinente: "acorde a los requerimientos de la época y del contexto, manteniendo una mirada crítica en torno a los asuntos sociales que determinan el quehacer educativo para buscar el beneficio para un mayor número de personas" (p. 12). 


\section{2. $\mathcal{E}$ strategia metodológica}

El trabajo se adscribe al paradigma constructivista que "se desarrolla a partir de la idea central de que no hay realidad objetiva que pueda ser descubierta. No se descubre sino se construye" (Tójar, 2006, p. 63). Desde este paradigma, las personas, en este caso docentes y estudiantes, tienen tras sí una historia singular de experiencias e interacciones que les permite "referirse a una misma realidad construyendo y reconstruyéndola de manera diferente" (p. 63). Se emplea un enfoque cualitativo con el propósito de comprender e interpretar la realidad, es decir, "entenderla en su globalidad, con todos sus elementos funcionando de forma conjunta... siempre dentro del contexto de referencia" (p. 151). En este sentido, la intención del trabajo es recuperar la experiencia de las IFAD a través de la voz del profesorado, en su rol de formador/a de formadores o estudiantes.

El ejercicio se centra en el análisis de las experiencias compartidas en dos eventos: el Encuentro Virtual de Instituciones Formadoras y Actualizadoras de Docentes (Secretaría de Educación y Deporte (SEyD), 2020a), realizado el 16 de noviembre de 2020, y; el Encuentro virtual de instituciones de posgrado. El trabajo docente no presencial en el periodo de contingencia sanitaria por el SARS-COV2 (SEyD, 2020b), llevado a cabo el 11 de diciembre del mismo año; ambos eventos fueron organizados por la SEyD y se encuentran disponibles en video en la plataforma YouTube. Para Gibbs (2012), los datos cualitativos provienen de fuentes diversas como entrevistas, observaciones, correos electrónicos, diarios y videos, dado que contienen múltiples formas "de comunicación —escrita, en audio o visual一 o comportamientos humanos, símbolos o artefactos culturales” (p. 23).

Las instituciones participantes se clasificaron según su función en el Cuadro 1.

\section{Cuadro 1}

Instituciones Formadoras y Actualizadoras de Docentes en el estado de Chihuahua

\begin{tabular}{|l|l|l|l|l|}
\hline INSTITUCIÓN & $\begin{array}{l}\text { MODALIDAD DE } \\
\text { FORMACIÓN }\end{array}$ & SEDES & $\begin{array}{l}\text { NO. DE } \\
\text { DOCENTES }\end{array}$ & $\begin{array}{l}\text { NO. DE } \\
\text { ESTUDIANTES }\end{array}$ \\
\hline $\begin{array}{l}\text { Centro Chihuahuense de Estudios de } \\
\text { Posgrado }\end{array}$ & $\begin{array}{l}\text { Superación } \\
\text { profesional }\end{array}$ & $\begin{array}{l}\text { Chihuahua, } \\
\text { Cuauhtémoc, Juárez }\end{array}$ & - & 370 \\
\hline $\begin{array}{l}\text { Centro de Actualización del } \\
\text { Magisterio }\end{array}$ & $\begin{array}{l}\text { Actualización } \\
\text { Formación inicial }\end{array}$ & Chihuahua, Juárez & 18 & 90 \\
\hline Centro de Investigación y Docencia & $\begin{array}{l}\text { Superación } \\
\text { profesional }\end{array}$ & Chihuahua, Juárez & 53 & 305 \\
\hline $\begin{array}{l}\text { Escuela de Trabajo Social del Estado } \\
\text { Profra. Guadalupe Sánchez de Araiza }\end{array}$ & Formación inicial & Chihuahua & - & 352 \\
\hline $\begin{array}{l}\text { Escuela Normal Experimental Miguel } \\
\text { Hidalgo }\end{array}$ & Formación inicial & Parral & - & - \\
\hline $\begin{array}{l}\text { Escuela Normal Rural Ricardo Flores } \\
\text { Magón }\end{array}$ & Formación inicial & Saucillo & 434 \\
\hline $\begin{array}{l}\text { Escuela Normal Superior Profr. José } \\
\text { E. Medrano R. }\end{array}$ & $\begin{array}{l}\text { Actualización } \\
\text { Formación inicial } \\
\text { Superación } \\
\text { profesional }\end{array}$ & $\begin{array}{l}\text { Chihuahua, Juárez, } \\
\text { Nuevo Casas } \\
\text { Grandes, Parral }\end{array}$ & 116 & 1828 \\
\hline Escuela Normal Yermo y Parres & Formación inicial & Creel & 12 & 102 \\
\hline
\end{tabular}




\begin{tabular}{|l|l|l|l|l|}
\hline INSTITUCIÓN & $\begin{array}{l}\text { MODALIDAD DE } \\
\text { FORMACIÓN }\end{array}$ & SEDES & $\begin{array}{l}\text { NO. DE } \\
\text { DOCENTES }\end{array}$ & $\begin{array}{l}\text { NO. DE } \\
\text { ESTUDIANTES }\end{array}$ \\
\hline $\begin{array}{l}\text { Institución Benemérita y Centenaria } \\
\text { Escuela Normal del Estado de } \\
\text { Chihuahua Profr. Luis Urías } \\
\text { Belderráin }\end{array}$ & Formación inicial & Chihuahua & - & 970 \\
\hline $\begin{array}{l}\text { Universidad Pedagógica Nacional del } \\
\text { Estado de Chihuahua }\end{array}$ & $\begin{array}{l}\text { Actualización } \\
\text { Formación inicial } \\
\text { Superación } \\
\text { profesional }\end{array}$ & $\begin{array}{l}\text { Camargo, } \\
\text { Chihuahua, Creel, } \\
\text { Cuauhtémoc, } \\
\text { Delicias, Guachochi, } \\
\text { Guadalupe y Calvo, } \\
\text { Juárez, Madera, } \\
\text { Nuevo Casas } \\
\text { Grandes, Parral }\end{array}$ & 4580 \\
\hline
\end{tabular}

Fuente: Elaboración propia con datos de la SEyD (2020a; 2020b).

El trabajo de campo se realizó en tres etapas siguiendo las pautas señaladas por Tójar (2006):

a. De organización: Esta etapa remite a la transformación en texto del material audiovisual para su análisis (Gibbs, 2012). En la transcripción, se distinguió la experiencia de las instituciones participantes, con énfasis en la perspectiva de personal directivo, docentes y estudiantes.

b. De análisis: Luego de la transcripción literal del encuentro, se realizaron dos tareas: 1. la revisión de datos, que consistió en "la constante y recurrente vuelta a las notas e informaciones recogidas con el fin de reflexionar sobre ellas, estudiarlas, complementarlas, buscar relaciones sincrónicas y diacrónicas" (p. 287), y; 2. la reducción de datos, que "consiste en una cierta simplificación de la información recogida, que pretende hacerla más manejable e interpretable eliminando lo superfluo y lo redundante... [sin] perder nunca la esencia, lo sustancial, de la información” (p. 287).

En esta fase se recurrió al análisis de contenido - que permitió realizar inferencias con base en el análisis de significados, significantes, contexto, condiciones de producción, entre otros elementos (Bardin, 2002) - , para codificar y sistematizar la información en correspondencia a los objetivos y preguntas de la investigación. Como estrategia de análisis, se emplearon los tres niveles del lenguaje propuestos por Ruiz (2006): nivel de superficie, se realizó con un análisis vertical de la experiencia de cada institución para describir las afirmaciones de los informantes; nivel analítico, a partir de un análisis horizontal, la información se clasificó, ordenó y categorizó alrededor de criterios de afinidad y diferenciación, y; nivel interpretativo, con un análisis transversal, el ejercicio se orientó a comprender el sentido de la información para resignificarla. Los tres niveles permitieron elaborar un sistema de categorías y subcategorías que, al ordenarse jerárquicamente y organizarse en conjuntos de significado, posibilitaron la redacción de los resultados.

c. De interpretación: En esta etapa se "pretende extraer una significación que profundice en los significados obvios o superficiales y recupere los significados ocultos o encubiertos que pueden dar sentido a la experiencia vivida" (Tójar, 2006, p. 104). Con tal propósito, se emplearon dos estrategias: la consolidación teórica, que permitió revisar la pertinencia de los datos en las categorías de análisis, y; la aplicación de otras teorías para comparar los hallazgos con diversos estudios u otros marcos analíticos. Como resultado, emergieron un conjunto de desafíos que, inicialmente, se organizaron en tres grupos según se tratara de actividades administrativas, docentes y de apoyo; luego de revisar los ocho 
retos identificados por Ortega (2020), en torno a procesos administrativos, permanencia, pertinencia, inclusión, cobertura, competencias tecnológicas, procesos educativos y atención socioemocional, el ejercicio analítico se redefinió a fin de denotar las particularidades de la realidad analizada.

La incorporación de las tecnologías digitales a las prácticas de investigación social ha generado la aparición de nuevos desafíos éticos, particularmente, ante la imposibilidad de establecer "normas fijas para orientar éticamente las decisiones en el estudio de fenómenos mediados por internet" (Estalella y Ardèvol, 2007, párr. 79). El trabajo se realizó a partir de las nociones de público/privado como criterio para definir la responsabilidad ética de los investigadores, y los principios de la ética situada propuesta por Allen (1996). En el caso concreto, los foros se colocaron en la plataforma digital como videos públicos para socializar los resultados del ejercicio académico (SEyD, 2020c). Para Estalella y Ardèvol (2007) "cuando la investigación se realiza en espacios públicos no es necesario solicitar permisos para realizar la investigación... esto se hace extensible a los medios de comunicación social" (párr. 20). Sin embargo, dado que la línea entre lo público/privado rebasa requisitos técnicos - como el uso de contraseñas y seudónimos-, se requiere de una ética situada que permita definir las consideraciones éticas - anonimato, privacidad/publicidad, sensibilidad de la información, entre otros-, en correspondencia a los propósitos de la investigación, los intereses de los informantes y las condiciones del contexto. Considerando que "la primera reflexión ética que el investigador debe realizar le obliga a procurar que sus acciones no perjudiquen a aquellos que forman parte de su estudio" (Estalella y Ardèvol, 2007, párr. 13), aun cuando se prescindió del consentimiento informado, la protección de los participantes se realizó tomando en cuenta el principio de paridad, que implicó tomar la participación de los informantes - directivos, docentes y estudiantes - con la misma importancia, y el principio de cautela en la emisión de juicios para evitar simplificar, exagerar u ocultar información (González et al., 2012).

\section{Resultados}

La presentación de los resultados se realiza siguiendo los ocho retos identificados por Ortega (2020); además, se incluyen citas textuales para hacer explícitas las voces y perspectivas de los participantes.

\subsection{Los procesos administrativos}

La contingencia sanitaria ha replanteado el funcionamiento de las IFAD y su capacidad para enfrentar la crisis, en particular, sus habilidades de gestión en apego a la filosofía institucional. Sus lemas, expresiones sintéticas de las aspiraciones colectivas, reflejan elementos identitarios comprometidos con procesos humanistas y de transformación.

Estamos para servir, como dice el lema de nuestra escuela: Hacer del magisterio una forma de servir a México. (coordinador, ENSECH, 1h03m14s) 
Tratamos de ser fieles a nuestro lema de "Formar profesionales de la educación con calidad y sentido humano"; es muy importante la calidad, pero, no es menos importante la relación humana con nuestros estudiantes... Hay clases que han sido más espacios de contención emocional..., hubo clases donde estaban desbordadas las emociones por parte de los estudiantes $\mathrm{y}$, bueno, tenemos que ser comprensivos, empáticos, aprender a caminar juntos. (director, CCHEP, 136m30s)

En los procesos de gestión de las IFAD apremia la respuesta a situaciones vinculadas con la implementación de plataformas digitales, la administración de los recursos financieros, la agilidad de los servicios de control escolar -inscripción, captura de calificaciones, entrega de constancias, control de asistencia-, entre otras.

¿Cómo nos organizamos para el trabajo a distancia? a. Atención a las disposiciones de las autoridades gubernamentales; b. Socialización y organización a partir de sus situaciones contextuales; c. Reuniones de trabajo en línea en múltiples espacios y academias; d. Atención de situaciones desde lo general hacia lo específico hasta las situaciones particulares; e. Movilizar los procesos de las áreas sustantivas: docencia, investigación, extensión y difusión universitarias (directora, UPNECH, 67m45s).

Ante la nueva realidad del trabajo a distancia, el enfoque empleado se centró en transformar los diferentes procesos y elementos que conforman la experiencia del estudiante... El proceso de inscripción se digitalizó...; Con la plataforma administrativa se ha dado atención oportuna a los estudiantes en cuanto a trámites de servicios escolares como: constancias, boletas, relaciones de estudios, titulación y expedición de credenciales, entre otros (director, CCHEP, 88m44s).

En este punto, Ortega (2020) señala que, frente al estrés institucional, las IFAD desarrollan una resiliencia específica y, gradualmente, se convierten en escuelas resilientes. Según Day y Gu (2016), las escuelas resilientes "son lugares en los que profesores y alumnos aspiran a su propio aprendizaje y desarrollo y en los que se comparte, se valora y se incorpora en la vida diaria un claro sentido del propósito moral" (p. 205). Desde esta perspectiva, el personal directivo tiene un papel clave en el aseguramiento de las "condiciones, estructuras y culturas de la enseñanza óptimas, capacitando a los profesores para que respondan positivamente a las inevitables incertidumbres cotidianas" (p. 224).

\subsection{2 a permanencia}

Otro reto es el de la permanencia: Ya están adentro, ahora ¿qué hacemos para que realmente no se desilusionen? (Ortega, 2020, 31m14s)

En general, el ingreso a la educación superior puede significar un quiebre en la trayectoria escolar de las y los estudiantes, en especial, entre aquellas/os que carecen de los capitales culturales, académicos y sociales necesarios para enfrentarla con éxito (López et al., 2018). En respuesta, las Instituciones de Educación Superior (IES) han alentado el establecimiento de programas de tutoría para contribuir a abatir los problemas de deserción, rezago y bajos índices de eficiencia terminal (Asociación Nacional de Universidades e Instituciones de Educación Superior (ANUIES), 2001). Sin embargo, en las 
escuelas normales "se carece de un modelo de acompañamiento para... el ingreso del estudiante, su permanencia y egreso" (Cruz y Delgado, 2019, p. 9).

No todos los estudiantes normalistas cuentan con dispositivos electrónicos o la conectividad a Internet en sus casas, por lo que se consideró establecer una comunicación cercana y empática con ellos... Más del 80\% tenían posibilidades de acceso a sus clases de manera sincrónica y sólo una minoría no podría hacerlo por situaciones como radicar en comunidades con dificultades de conectividad a Internet o, debido a que se compartían equipos entre los miembros de la familia. (docente, IByCENECH, 32m00s)

Me encuentro frente a un grupo de primer semestre... Tuve a mi cargo alumnos que iniciaban su vida universitaria, alumnos que tendrían expectativas sobre cómo es la universidad, alumnos que soñaban con entrar a las aulas de nuestra institución a recibir sus clases. Así que comencé a pensar en cuáles serían las necesidades que ellos tendrían, sobre todo, en pensar en qué alternativas tendría, como docente, para impartirles mi asignatura y generar en ellos el amor por aprender. (docente, ETS, 2h10m14s)

Frente a este escenario, Day y Gu (2016) señalan:

En vez de preguntarnos: ... “¿Cómo podemos hacer que los profesores se queden en el centro?” ... Creemos que las cuestiones adecuadas son las siguientes: “¿Cómo podemos promover la resiliencia de modo que los profesores puedan disponer siempre de mejores oportunidades para esforzarse por enseñar [y aprender] de manera óptima cada día?” y “QQué tipos de formación, apoyo, entorno de trabajo, cultura, liderazgo y prácticas de gestión facilitarían dicho desarrollo? (pp. 40-41)

Para Murillo y Duk (2020), la pandemia derivó en la interrupción del aprendizaje, en la presión para que madres y padres dirigieran la enseñanza a distancia, en la carga extra de trabajo para el profesorado y, sobre todo, en el incremento de las tasas de abandono escolar. Al respecto, se advierte que, el logro de una experiencia de aprendizaje en línea significativa requiere de cercanía docente, reconocimiento de las emociones en el aprendizaje, ajustes razonables y dispositivos pedagógicos centrados en las necesidades de las y los estudiantes (Díaz-Barriga y Barrón-Tirado, 2020).

Pensando en la vulnerabilidad que tienen nuestras estudiantes, al presentar desventaja por ser mujeres y encontrarse en comunidades geográficas con algunas limitantes de accesibilidad y conectividad, se realizaron algunos ajustes respecto al tiempo sincrónico de atención a los cursos de la malla curricular; pensando en el bienestar y, con la intención de brindar un mejor servicio, se ofrecen asesorías grupales e individuales para quien lo necesite a través de plataformas que sean de mayor facilidad para ellas. Como un compromiso hacia nuestras alumnas también se les atiende a través de la Unidad de Atención Integral al Estudiante Normalista para abatir los posibles sesgos de su formación personal y profesional. (subdirectora, ENRS, 1h06m07s) 
Se grabaron las asesorías de clase, cuyas ligas se enviaban al alumnado para que puedan revisar los videos de lo visto en clase. Al estudiantado que presenta problemas de aprovechamiento, se le ha asignado un tutor para dar seguimiento y apoyo académico. Actualmente, se han dispuesto espacios como el laboratorio de informática en los que se han implementado las medidas de sana distancia y de prevención del contagio para que el alumnado con carencias económicas, que no cuenten con Internet o con equipo de cómputo, no dejen sus estudios y puedan continuarlos asistiendo a la institución educativa bajo todas las medidas de salud. (docente, ETS, 2h03m53s)

Para compensar las limitaciones de los alumnos con dificultades de acceso se realizaron las siguientes actividades: llamadas telefónicas, flexibilidad con las fechas y horarios para la entrega de trabajos y tareas, adecuaciones curriculares para los que no tienen conectividad, disponibilidad en horarios de atención, seguimiento continuo a los alumnos, comunicación continua y diálogo. (docente, ENYP 2h29m28s)

\section{3. $\mathcal{L}_{\text {a pertinencia }}$}

En sentido amplio, la pertinencia refiere a la "satisfacción de las necesidades del campo profesional, oportunidad, adecuación y conveniencia de los programas educativos en relación con las expectativas y la evolución de las necesidades y prioridades... a escala local, regional y nacional" (Comité para la Evaluación de Programas de Pedagogía y Educación (CEPPE), 2015, p. 7). En el ámbito de la formación docente, la pertinencia alude a la atención a las características del contexto, las necesidades específicas de los docentes y las condiciones de la escuela (Santibáñez et al., 2018).

[Entre los docentes necesitamos] abatir la resistencia al cambio, desarrollar habilidades digitales, diseñar planeaciones u objetos de aprendizaje acordes a esta nueva modalidad, adaptar la enseñanza al contexto sociocultural. (docente, IByCENECH 47m00s)

En el contexto de la pandemia, una de las cuestiones centrales refirió a distinguir los contenidos disciplinares más relevantes, sobre todo porque éstos comúnmente son "excesivos..., alejados de la realidad de los educandos y sus comunidades" (Díaz-Barriga y Barrón-Tirado, 2020, p. 2). Frente a esta tarea, las academias se consolidaron como espacios formales para que docentes, directivos y personal de apoyo reflexionaran sobre el trabajo, planificaran estrategias y organizaran acciones. Estos espacios de trabajo colectivo pueden contribuir a impulsar "procesos sistemáticos de reflexión, innovación, solución conjunta de problemas y formación de redes para el intercambio de conocimientos" (INEE, 2018a, p. 65), sobre todo, porque en las IFAD "prevalecen culturas escolares que conciben a la enseñanza como una práctica individual [que] afecta el poco desarrollo de estrategias de aprendizaje entre pares” (INEE, 2018b, p. 4).

Un principal elemento para la organización de este trabajo han sido las academias con las y los profesores, con los coordinadores de los programas de Maestría y Doctorado para el intercambio de experiencias que permiten la mejora. (directora, UPNECH, 58m26s) 
Sobre la marcha, la institución y los propios docentes reconocimos que se había gestado un nuevo lenguaje, con el que no estamos lo suficientemente familiarizados. Encontramos dificultades conceptuales que aun precisan diálogo, reflexión y discusión, mismas que hemos abordado en las academias de materia, trayecto formativo o por semestre. (directora, CAM, 2h40m00)

La Covid-19 transformó los contextos de implementación del currículo, no solo por el uso de herramientas y plataformas virtuales y la necesidad de contextualizarlas para asegurar la pertinencia de los contenidos, sino porque requirió priorizar aprendizajes y competencias indispensables en la actual realidad. Así, ante el carácter disruptivo de la emergencia sanitaria se gestó un nuevo modelo educativo. En este punto, Vezub (2020) alude a la capacidad de las y los docentes para modificar o adaptar estrategias ante el cambio en las variables del contexto de trabajo, del alumnado, del escenario, de las formas de vincularnos, de las formas de enseñar, entre otras.

Otro [reto] es el de la pertinencia: ¿qué contenidos abordar? ¿qué es lo más relevante en este momento? ¿qué es lo que importa cuando se están perdiendo vidas, cuando se están perdiendo empleos, cuando hay familias que están en condiciones terribles? (Ortega, 31m27s)

La emergencia ha puesto en crisis una práctica docente estable..., esto nos permite hacer una serie de reflexiones: primero, sobre qué es importante enseñar, aprender; sobre el tipo de práctica docente que tenemos que privilegiar en condiciones de emergencia... Habrá que discutir la parte pedagógica, didáctica en el contexto de la educación a distancia: qué es lo más importante enseñar, cómo enseñarlo, qué tipo de materiales, qué tipo de estrategias didácticas, qué tipo de actividades y cuáles son los mejores medios de entrega. (director, CCHEP 4h03m47s)

El problema surgió, luego de las primeras sesiones, cuando docentes y estudiantes "comenzaron a reportar experiencias poco significativas en la virtualidad, la carencia de habilidades para el aprendizaje autónomo, la ausencia de mediación docente, el exceso de tareas y actividades repetitivas y poco motivantes" (Díaz-Barriga y Barrón-Tirado, 2020, p. 3); así la pertinencia rebasó los aspectos curriculares y se centró en la capacidad del profesorado para adaptar las actividades a las necesidades, estilos de aprendizaje y hábitos de estudio de las y los estudiantes, a través de estrategias de acompañamiento oportunas y permanentes. "Lo deseable es que sean currículos flexibles, de código abierto, situados, que permitan la construcción de trayectorias personales para aprender [en la estabilidad y en la incertidumbre]” (p. 4).

Al iniciar mi planeación me realicé diferentes interrogantes: ¿Cómo dar a conocer los temas de mi materia? ¿Cómo elaborar dinámicas para las reuniones virtuales? ¿Cómo ser empática con mis alumnos y poder conocerlos más? Esto me llevó a pasar bastantes días investigando cómo mejorar la reunión, aprender sobre nuevas herramientas tecnológicas a las que no me había abierto a la posibilidad... Es cierto, requerí salir de mi zona de confort y aventurarme a nuevas posibilidades para que, tanto mis alumnos como yo, pudiéramos disfrutar de nuestras clases, aprendiendo juntos. Tuve que cambiar mi modo de pensar. (docente, ETS, 2h11m28s) 
Estoy convencido que la discusión es más de carácter didáctico, académico, que de tecnologías. Muchas escuelas cometieron el error de que cuando se vino este asunto le hablaron al de sistemas y le dijeron, necesitamos educación a distancia. La educación no es un asunto de ingenieros, es un asunto de educadores. (director, CCHEP, 4h10m14s)

\subsection{Za inclusión}

En el modelo educativo para la educación obligatoria (SEP, 2017), se define a la educación inclusiva como aquella que:

garantiza el acceso, permanencia, participación y aprendizaje de todos los estudiantes, con especial énfasis en aquellos que están excluidos, marginados o en riesgo de estarlo. Esto se realiza mediante la puesta en práctica de un conjunto de acciones orientadas a eliminar o minimizar las barreras que limitan el aprendizaje y la participación. Dichos obstáculos pueden surgir de la interacción entre los estudiantes y sus contextos: las personas, las políticas, las instituciones, las culturas y las prácticas. (p. 205)

En la realidad de las IFAD, alrededor de la educación inclusiva se observan dos procesos: la formación docente para la inclusión y la formación docente en la inclusión. El primer término, refiere a los aspectos curriculares considerados en las diferentes modalidades de la formación docente para generar entre el profesorado - en formación o en servicio - actitudes positivas ante la diversidad, así como las competencias para diseñar ambientes de aprendizaje inclusivos, en apego a los principios de equidad e igualdad; mientras, el segundo alude a las prácticas inclusivas que docentes, personal directivo y administrativo realizan frente al estudiantado en situación de vulnerabilidad en un marco de respeto, aceptación, empatía y solidaridad. Para Flores et al. (2017) las prácticas inclusivas refieren a las acciones del profesorado para lograr el desarrollo integral de todos sus estudiantes en función de las necesidades del grupo y el reconocimiento de la diversidad como elemento inherente a los procesos de formación.

Una de las características principales de la institución radica en su sistema de internado para jóvenes mujeres de escasos recursos económicos y de pueblos originarios. (subdirectora, ENRS, 1h04M31s)

De acuerdo con Murillo y Duk (2020), la emergencia derivada por la Covid-19, y la consecuente suspensión de toda actividad educativa presencial, afectó particularmente a las y los más vulnerables: las y los estudiantes en situación de discapacidad, de familias con menor nivel socioeconómico, migrantes, indígenas, entre otros; para quien la emergencia sanitaria gradualmente se convierte en una emergencia social. Así, se excluye a todos aquellos que "no tienen las destrezas o no están preparados en el uso de los dispositivos digitales" (p. 12). En esta línea, Díaz-Barriga y Barrón-Tirado (2020), afirman que "el sistema educativo en general, y las estructuras curriculares en particular, operan en nuestro contexto de manera inequitativa, no inclusiva, contraviniendo los preceptos de la justicia social y curricular" (p. 2). 
En la totalidad de las IFAD se han establecido formas de continuidad académica a través de diversas herramientas tecnológicas. En conjunto, la experiencia institucional revela la implementación de modalidades de educación en línea por Internet en donde destaca el uso de plataformas virtuales de aprendizaje asincrónico - Moodle, Google Classroom, Edmodo-, clases en vivo -Zoom, Google Meet-, mensajería instantánea - WhatsApp, Facebook—, y correo electrónico; mientras, en la modalidad fuera de línea se incluye la elaboración de materiales impresos, guías de trabajo y llamadas telefónicas.

Otro elemento más que hemos tenido es la organización de los programas educativos en la plataforma Moodle que permite desarrollar los contenidos en tiempos no reales precisamente o que no conllevan al uso de una conectividad permanente como lo requiere el Zoom o Google Meet como herramientas complementarias que se han utilizado para tener esa cercanía, al menos cara a cara a través de la cámara, que permiten el debate de ideas, análisis y retroalimentación. (directora, UPNECH, 58m56s)

Las sesiones de los programas de maestría y doctorado se han llevado de acuerdo con lo programado en cada una de las semanas mediante el uso de plataformas de videoconferencia como son Zoom, Meet y Jitsi. Asimismo, hemos hecho uso de plataformas de atención asincrónica como Moodle y Classroom. El trabajo se ha complementado con el uso de WhatsApp, correo electrónico o incluso llamadas telefónicas, además del uso de formularios de Google para ayudarnos en el trabajo de campo. (subdirector, CID, 78m35s)

Previo a la pandemia, en México los índices de pobreza y de pobreza extrema habían aumentado, develando la persistencia de las desigualdades y el creciente descontento social (OREALC/UNESCO Santiago, 2020, p. 6). Con todo, la Covid-19 visibilizó el acceso desigual a las tecnologías, situación que exigió de las IFAD priorizar los esfuerzos dirigidos a mantener el contacto cercano con el estudiantado, en especial, con aquellos que radican en regiones con mayores dificultades de conexión y se encuentran en condiciones socioeconómicas desfavorables.

En la región de Creel, las condiciones críticas impiden el acceso a alguna red de Internet, es por lo que la Escuela Normal Superior ha impulsado el trabajo a distancia con esos alumnos que se encuentran en situación de desventaja, creando redes de comunicación asertiva con ellos, brindando opciones de trabajo académico constante, pero, sobre todo, desarrollando la empatía y la solidaridad ante esta situación que prevalece en el entorno serrano del estado de Chihuahua. La falta de conectividad y el acceso se vuelven problemas muy intermitentes, por tal motivo las sesiones que se llevan a cabo en línea son aprovechadas en su totalidad para que esos estudiantes que ya tienen que pagar hospedaje, alimentación y traslados de comunidades a otras, tengan contacto con el conocimiento y no sea en vano el sacrificio que están realizando para profesionalizarse en la práctica docente y en la mejora de su desarrollo profesional. (docente, ENSECH, 49m42s) 


\subsection{La cobertura}

Para responder a la emergencia sanitaria, las IFAD han optado por la continuidad académica mediante recursos en línea. Hoy, Internet constituye la principal opción para vincular a la escuela con los estudiantes en condiciones de confinamiento. Según la OREALC/UNESCO Santiago (2020),

aunque la región ha avanzado de manera significativa en la reducción de las brechas de acceso al mundo digital en los últimos años, particularmente gracias a la masificación de la conectividad móvil, aún persisten brechas considerables en el acceso efectivo al mundo digital, lo que tiene profundas implicaciones en las oportunidades y la participación de las nuevas generaciones. (p. 5)

Según lo declarado por las y los participantes, el estudiantado continuó sus estudios durante la contingencia, salvo aquellas personas que ante diversas circunstancias abandonaron los programas.

[De nuestros estudiantes] 74\% viven o trabajan en el medio urbano, el resto en el medio rural. El 96\% no presentan problemas de conectividad, el 59.5\% no presenta problema para empezar las aulas Meet, el 49.5 algunas veces (docente, ENSECH, 44m10s).

El perfil de nuestros estudiantes es muy diverso y las carencias académicas de todo tipo son muy frecuentes... La mayoría proviene de familias de escasos recursos económicos y el 16\% es de origen indígena. (docente, ENYP, 2h17m51s)

De acuerdo con la información presentada, la mayoría de las y los estudiantes de las IFAD tienen acceso a Internet y a una computadora en el hogar, recursos elementales para el aprendizaje virtual, sin embargo, insuficientes "porque no todas las modalidades ofrecen las mismas oportunidades de uso y aprovechamiento, ya que estas también dependen en gran medida de la calidad de la conexión y el tipo de dispositivo" (OREALC/UNESCO Santiago, 2020, p. 6).

El 96\% de los alumnos vive en zonas rurales, esto representa un problema para el trabajo a distancia porque la conectividad es escasa o nula y muy deficiente... El 75\% de los alumnos usan datos para elaborar sus trabajos, para videollamadas, para usar el WhatsApp, el correo electrónico o plataformas, esto representa un problema serio porque no se les puede exigir de una manera intransigente cuando se tiene conocimiento de lo que implica estar adquiriendo datos; el 95\% de los alumnos tienen computadora. (docente, ENYP, 2h18m40s)

Creo que las subjetividades han sacado a la luz múltiples problemáticas, ha modificado nuestra experiencia inmediata de ser y estar en el mundo. Esto lo he aprendido de los estudiantes, desde aquellos que corrieron a buscar trabajo, desde aquellos que se han volcado a las tecnologías, desde aquellos que han comprado y se han endrogado para seguir cumpliendo. (directora, CAM, 4h35m46s) 
Aun cuando, en general, el estudiantado cuenta con conectividad, existen alumnos/as que permanecen excluidos, en especial en las regiones serranas y en las regiones urbanas con vulnerabilidad económica. Esta problemática se ha hecho evidente a nivel internacional, por ejemplo, en España, se ha considerado "que si un porcentaje de alumnos, por pequeño que sea, no puede acceder a las herramientas de educación online se rompe el principio de igualdad educativa” (CAF, 2020, p. 22). Esto hace necesario, asegurar las condiciones básicas entre las poblaciones menos favorecidas, particularmente, porque algunos estudiantes refieren utilizar Internet móvil a través de planes de prepago que proporcionan servicio limitado para navegar o utilizar las plataformas de aprendizaje y otras vías que se están empleando para la continuidad de los estudios. Sobre el punto, "se espera que el reconocimiento del acceso a internet como un derecho humano plasmado en la Constitución pueda aumentar el porcentaje de personas con este servicio" (MEJOREDU, 2020a, p. 66). Sobre todo, porque "no se trata solo de una diferencia de acceso a equipamiento, sino también del conjunto de habilidades que se requieren para poder aprovechar esta oportunidad" (OREALC/UNESCO Santiago, 2020, p. 7).

Además, la falta de equipo de cómputo y conexión a Internet se extiende al profesorado. La necesidad de dispositivos digitales para docentes es un reclamo internacional, sobre la que se han llevado a cabo distintas iniciativas. En El Salvador, en el marco de la digitalización de la educación, se ha propuesto la adquisición de equipo tecnológico para el 100\% del profesorado del sector público; mientras, en Argentina se gestiona el ofrecimiento de créditos bancarios a una tasa subsidiada para que docentes en general puedan comprar computadoras (OREALC/UNESCO Santiago, 2020).

Esta emergencia ha puesto de manifiesto las carencias y desigualdades tanto en la disponibilidad de recursos como en la preparación de docentes y estudiantes para transitar hacia las modalidades de educación a distancia... Es necesario reconocer la diversidad de los docentes porque no todos tenemos las mismas condiciones económicas para solventar los gastos que surgen al tener que buscar otras alternativas de conectividad o costearnos un buen equipo de cómputo para cumplir con nuestro trabajo, sin dejar desprotegidas a nuestras familias económicamente. Se tiene que reconocer el esfuerzo de quienes salen de casa en busca de un cibercafé para impartir la clase o asistir a alguna academia virtual. Se tiene que reconocer que los docentes también son padres y madres de familia que, además, comparten horarios de clase con sus hijas e hijos en un mismo espacio, utilizando la misma señal de Internet, presentando fallas al transmitir sus clases, por tanto, nuestra demanda es el servicio gratuito de Internet para docentes y estudiantes durante la pandemia. (Docente, ENRS, 1h13m05s)

\section{6. $\mathcal{L}$ as competencias tecnológicas}

Para Murillo y Duk (2020):

la irrupción de la educación a distancia producto de la emergencia, ha dejado al descubierto la brecha digital existente entre las escuelas y sus docentes. Profesores que manejan las tecnologías con sentido pedagógico, que saben cómo diseñar procesos de aprendizaje en entornos virtuales, desafortunadamente son la minoría. (pp. 12-13) 
Cada IFAD precisó construir mecanismos para la profesionalización de sus estructuras docentes a fin de responder pertinentemente a las necesidades de sus estudiantes. Dado que las nuevas condiciones han requerido que el profesorado utilice plataformas virtuales con las que no necesariamente se encontraba familiarizado, todas las instituciones consideraron entre sus estrategias el diseño de trayectos de formación para capacitar y actualizar a sus docentes en el uso y manejo de las Tecnologías de la Información y la Comunicación (TIC). Cabe destacar que la formación tecnológica constituye, al menos desde 1994, uno de los ejes de acción pública para el desarrollo profesional docente (INEE, 2018a).

Sobre el trabajo a distancia ante Covid-19... No estábamos preparados, teníamos alguna experiencia, pero no suficiente, menos como única alternativa. Nos vimos obligados a emprender la marcha, ahora con estas nuevas condiciones para tratar de sortear la situación y mantener a flote el servicio educativo con normalidad en una nueva normalidad... La pandemia nos orilló a sumarnos a un mundo algo desconocido, esta condición generó necesidades y propició la exploración de dispositivos, instrumentos, tecnologías, técnicas y estrategias de enseñanza-aprendizaje, de comunicación, de interacción en ambientes virtuales, etcétera. Con todo, hoy podemos decir que ha resultado muy enriquecedor; trabajar a distancia se ha convertido en una experiencia gratificante. (director, CID, 73m15s)

De manera virtual se ha dado seguimiento a los procesos de capacitación y formación docente. Nuestra planta docente se ha actualizado a través de la capacitación y acompañamiento para el uso del aula virtual Zoom y la plataforma educativa Moodle. (docente, CCHEP, 94m18s)

En algunas instituciones, los recursos y plataformas digitales son habituales - ya sea como herramientas complementarias para el trabajo presencial o como características inherentes a sus programas-, por ejemplo, el Centro de Estudios y Educación Virtual (CEEV) de la UPNECH y la Maestría en Educación para el Desarrollo Profesional Docente de la ENSECH.

El programa considera el desarrollo de clases en línea de lunes a viernes con una sesión al mes de manera presencial, lo que ha favorecido la rápida adaptación del alumnado y catedráticos a la nueva forma de trabajo provocada por el confinamiento. (docente, ENSECH, 42m02s)

Según la Organización de las Naciones Unidas para la Educación, la Ciencia y la Cultura (UNESCO, 2019), ser un docente competente en el ámbito de las TIC, refiere a la movilización de 18 competencias organizadas alrededor de seis aspectos de la práctica profesional -comprensión del papel de las TIC en las políticas educativas, currículo y evaluación, pedagogía, aplicación de competencias digitales, organización y administración, y aprendizaje profesional docente-, que se materializan en la adquisición, profundización y creación de conocimientos, donde la formación docente, el acompañamiento pedagógico y técnico tienen un papel clave. En este punto, un participante comentó: 
También es cierto que ha habido ciertas dificultades, por ejemplo, en algunos alumnos o docentes que no han desarrollado totalmente o a cabalidad en un mayor nivel el manejo de los recursos tecnológicos ha significado un gran reto en el cual hemos avanzado. (docente, UPNECH, 59m43s)

\subsection{Los procesos educativos}

Para Ortega (2020), un eje de tensión "fue el manejo de las metodologías de enseñanza-aprendizaje en línea: ¿cómo superar las estrategias tradicionales expositivas para favorecer aprendizajes más auténticos, más situados, más pertinentes?" (32m26s).

Esta convulsa realidad interpeló a resignificar los procesos de enseñanza y aprendizaje. El currículo escolar, sus fines, objetivos, contenidos, métodos didácticos y evaluación, se pusieron en duda:

¿se estaba haciendo un empleo pedagógico y con sentido de tales tecnologías digitales o sólo un tratamiento técnico para poner en pantalla textos e imágenes en un esquema comunicativo unidireccional?... Quedó en evidencia que, a pesar del gran esfuerzo por trasladar el currículo a la virtualidad, no era cuestión de un [simple] ajuste a la modalidad en línea. (Díaz-Barriga y Barrón-Tirado, 2020, p. 2)

La pandemia nos obligó a dimensionar y trascender la función docente en esta nueva modalidad; construir nuevos procesos académicos con bastante interacción, además, ampliar nuestra disposición al trabajo individual, colectivo y más flexible... Se requiere de otra actitud, ocuparse de otra forma, pero también de propuestas innovadoras por parte de los docentes [para] evitar la monotonía en el desarrollo de las sesiones. (director, CID, 75m21)

Creo que hay que hacer un giro pedagógico...; estamos obligados a repensar problemas como el de una pedagogía que no parta de las desigualdades de la inteligencia, de una política que no niegue el disenso. (directora, CAM, 4h36m24s)

A través del territorio estatal, el profesorado ha implementado diversas acciones para adaptar los procesos educativos a los nuevos requerimientos. De forma individual o colectiva, se han elaborado materiales, diseñado cursos, planificado situaciones de aprendizaje, reorientado estrategias de enseñanza que, en conjunto, revelan el papel clave de las y los docentes, su iniciativa, creatividad y capacidad para trabajar en colaboración y en circunstancias adversas.

Desde el Gobierno Federal, se apela a la creatividad del magisterio en el diseño de estrategias para continuar con los procesos educativos en condiciones de crisis:

Esto implica ensayar y perfeccionar nuevas formas de enseñanza mediante tecnologías diversas y la necesidad de crear relaciones afectivas y pedagógicas distintas. Muchos docentes enfrentan el reto en medio de diversas limitaciones de acceso a estas tecnologías; otros, como quienes trabajan en escuelas multigrado o atienden a población migrante, afrontan un desafío aún mayor. (MEJOREDU, 2020b, p. 8) 


\subsection{2 a atención socioemocional}

A los procesos formativos se sumó el dolor, la angustia y la desesperanza. Expresiones como: "en los grupos que atendemos es múltiple la sensación de dolor", se hicieron presentes, por lo que fue necesario fortalecer conductas de empatía, solidaridad, tolerancia, resiliencia y no discriminación, coherentes con el carácter humanista de la educación.

Aflora la resiliencia, tanto de los alumnos como de los docentes, para lidiar con esta situación de emergencia sanitaria, soportando con entereza el confinamiento obligado y administrando la angustia de un futuro incierto, así como asumiendo lamentablemente el dolor de las pérdidas. (director, CID, 76m59s)

Tenemos que, en primer lugar, reconocer a los héroes de la salud que están allí todos los días atendiendo el problema de la contingencia sanitaria; en segundo, a los héroes de la educación, a todas las maestras, a todos los maestros que están hiperestresados porque los hemos saturado... porque hemos tenido fallecimientos y enfermedades cercanos. (director, CCHEP, 4h15m28s)

Docentes y directivos han enfrentado, en la medida de sus posibilidades y sin formación para ello, las demandas de apoyo socioemocional y de salud mental del estudiantado. Según la SEP (2017b) los procesos formativos tradicionalmente han priorizado el desarrollo de habilidades académicas desplazando la educación socioemocional, "porque hasta hace poco se pensaba que esta área correspondía más al ámbito educativo familiar que al escolar, o que el carácter o la personalidad de cada individuo determinan la vivencia y la expresión emocional” (p. 517).

Dentro de estas consecuencias encontramos que el trabajo a distancia ha provocado en los alumnos de la maestría: el 44.5\% estrés, el 11\% dolor de cabeza, el 9.2\% depresión, el 9.2\% ansiedad y $8.1 \%$ cansancio... Nos preocupamos por mantener un estado emocional estable en nuestros estudiantes, sabemos que esta situación ha causado desconcierto e incertidumbre. (docente, ENSECH, 45m33s)

Al dolor se suma la sobrecarga de actividades como detonante de estados de ansiedad y estrés, dos de los principales problemas de salud entre el magisterio, situación que aumenta al carecer de estrategias para compensar el cansancio y recuperar la fortaleza (Cervantes, 2020). En este sentido, se precisa de acciones para fortalecer los aspectos socioafectivos del aprendizaje.

Otro de los grandes retos es el trabajar bajo un esquema de educación a distancia ya que al estar en nuestra casa, la carga de trabajo se hace mayor porque al no saber cómo enfrentar esta pandemia las autoridades escolares nos están dejando a nosotros como docentes todo el trabajo administrativo... como maestros se nos está haciendo una carga de trabajo mucho mayor y como estudiantes tenemos que entregar trabajos, estar en las clases y todo eso y es muy pesado porque... "somos estudiantes y a la vez docentes frente a grupo". (Estudiante, UPNECH, 60m53s) 
Con frecuencia, las “mujeres, madres, estudiantes de programas de posgrado [se encuentran], bajo una presión constante por el establecimiento de fechas límite y la entrega de avances de ese trabajo de tesis por secciones, las cuales se evalúan semestralmente" (Cortazar y Aguirre, 2021, p. 250). Al respecto, una estudiante comenta:

En lo personal tuve que reorganizar mi vida familiar, laboral, tuve que aprender a gestionar y administrar mi tiempo para sacar los trabajos y las tareas pendientes... Por otro lado, están los inconvenientes que se presentan externos a uno que uno no puede solucionar "que el Internet está muy lento", "que la computadora se traba", "que no quiso prender", "que se descompuso", son agentes que uno no puede controlar y causan frustración. También está el cansancio mental, el cansancio visual... se cansan los ojos de estar tanto tiempo en la computadora y, al final del día, al final de la semana, termina uno cansado, frustrado, estresado. Ha sido un proceso muy difícil con una adaptación muy lenta la que hemos llevado. En lo personal tuve que cambiar mis hábitos de socializar, de aprender, de hacer mis trabajos, de trabajar individualmente, de autogestionar mis conocimientos, entre otras cosas. (estudiante, UPNECH, 64m52s)

Para el profesorado, el periodo de adaptación a la educación a distancia se ha traducido "en un conjunto de responsabilidades y exigencias que aumentan significativamente el tiempo de trabajo que las y los docentes requieren para preparar las clases, asegurar conexiones adecuadas y hacer seguimiento a sus estudiantes en formatos diversos" (OREALC/UNESCO Santiago, 2020, p. 10).

Los docentes nos hemos descubierto inmersos en un desgaste físico, mental y económico al incrementarse las horas de trabajo, de sufrir la invasión a nuestra privacidad, que además nos obligó a sacrificar espacios y horarios destinados a nuestras familias. (docente, ENRS, $1 \mathrm{~h} 13 \mathrm{~m} 55 \mathrm{~s})$

Los docentes expresan que la responsabilidad y las exigencias han aumentado, a la par del estrés y la carga de trabajo. (docente, ENYP, 2h25m45s)

En opinión de las y los participantes, existe una sobrecarga laboral respecto a las condiciones de trabajo anteriores a la pandemia, en especial entre las profesoras, quienes señalan dificultades para equilibrar la vida laboral con la vida personal. Situación que cobra relevancia al considerar que, en el pasado:

las mujeres docentes debían enfrentar jornadas laborales dobles, que incluían no solo su trabajo en el aula, sino también las labores docentes fuera de ella (tareas administrativas, planificación y preparación de clases, entre otras), así como el trabajo doméstico y de cuidado no remunerado. (OREALC/UNESCO Santiago, 2020, p. 10)

El dolor, la duda y el miedo se han hecho presentes también en la vida de las y los formadores de docentes. 
Nuestros maestros y estudiantes se han visto afectados por esta crisis: primero, en lo personal, por ejemplo: de los maestros de nuestra institución - considerando tanto a los contratados como los de base-, el 50\% de los que se aplicaron una prueba de Covid-19 resultaron positivos; el 39\% reporta haber estado enfermo de Covid-19; el 87\% de quienes reportan haber estado enfermos dicen haber tenido síntomas moderados, sin embargo hay un $12.5 \%$ que reportan haber estado graves; el 36\% reporta el fallecimiento de algún familiar como producto del Covid-19, el 57\% reporta el fallecimiento de algún amigo o compañero de trabajo, el $95 \%$ dice tener al menos un conocido que ha fallecido producto de esta crisis de salud y el 62\% tener algún enfermo de Covid-19 en su núcleo familiar. Esto nos habla del tamaño de la dimensión de la crisis no sólo educativa, sino la crisis personal, de la crisis humana que enfrenta la sociedad mexicana. (director, CCHEP, 129m49s)

Ante la carencia de certezas, la propuesta colectiva es repensar la educación para vivir y aprender en tiempos de crisis e incertidumbre. En este aspecto, Day y Gu (2016) proponen la noción de resiliencia en lo cotidiano, entendida como la capacidad docente para mantener aptitudes de deseo, determinación, energía y conocimiento frente a los cambios en las condiciones externas e internas de trabajo, modificaciones en el contexto o los efectos de acontecimientos personales imprevistos.

Cuando volvamos a la presencialidad ya no seremos los mismos porque habremos, primero, puesto en juego lo que es enfrentar la incertidumbre; creo que no hay nada más educativo, para cualquier persona de cualquier nivel, que enfrentarse a lo desconocido y tener que encontrar los medios para salir adelante. (director, CCHEP, 133m16s)

\section{Conclusión}

Luego de la cancelación de las actividades presenciales, la necesidad de continuar los procesos educativos generó desafíos que las IFAD han atendido mediante diferentes alternativas de adaptación, priorización y ajuste que implicaron cambios en la dinámica institucional. Las experiencias demuestran la presencia de iniciativas en los ocho retos analizados: procesos administrativos, permanencia, pertinencia, inclusión, cobertura, competencias tecnológicas, procesos educativos y atención socioemocional. Las acciones implementadas por las IFAD confirman la idea de que, la formación docente se reconfigura ante la Covid-19, mediante una serie de cambios en los procesos académicos, administrativos y organizacionales de cada institución. La experiencia institucional devela necesidades históricas y actuales que precisan atención, sin embargo, la pandemia se constituye en una oportunidad para impulsar un giro pedagógico en los procesos formativos en el estado de Chihuahua.

Este momento de crisis abre la oportunidad de construir nuevos ambientes educativos, otra mirada de educabilidad y la firme intención de promover la innovación disruptiva de estructuras y prácticas curriculares. No se trata de mejorar lo ya existente, sino de 
atreverse a cambios de fondo, en estructuras, procesos de gestión, posicionamiento de los actores de la educación. La innovación disruptiva requiere cambiar mentalidades y prácticas, un proceso de destrucción creadora, el arribo a nuevos paradigmas. (Díaz-Barriga y Barrón-Tirado, 2020, p. 2)

Del ejercicio se concluye que, según lo declarado por autoridades, docentes y estudiantes de las IFAD en la región, el periodo de contingencia sanitaria ha derivado un conjunto de experiencias y aprendizajes favorables para atender al magisterio chihuahuense. En el balance, se destaca el papel del profesorado, que ha redoblado esfuerzos para sobrellevar los desafíos, adaptarse a las circunstancias e innovar con los recursos disponibles. Sin duda, la contingencia ha afectado de diferente manera y con distinta intensidad a cada institución. Con todo, las IFAD precisan respuestas innovadoras y pertinentes ante los momentos de estabilidad e incertidumbre, para ello, resulta oportuno recuperar las experiencias de otras instituciones e interpelar especialmente a estudiantes y egresados. El uso de las plataformas y recursos educativos virtuales representó una alternativa para la continuidad académica de las IFAD. Pese a los logros, la experiencia analizada devela el relativo éxito del uso de las plataformas virtuales ante dos condiciones básicas: la conectividad y las competencias docentes. Así, la falta de acceso a equipos y conexión a Internet y la mínima alfabetización digital entre docentes y estudiantes, en especial, en aquellos con menor exposición a tales tecnologías, representan dos asuntos pendientes.

Entre las limitaciones del estudio destaca la nula intervención de los investigadores en la selección de los participantes, el empleo de los videos como fuente exclusiva para la recolección de datos y, considerando que los encuentros fueron convocados por las autoridades educativas, es probable que la información compartida por los participantes enfatice los logros y minimice las dificultades, lo cual pudo derivar sesgos interpretativos que trataron de resolverse con la revisión de fuentes secundarias. Sin embargo, los resultados del estudio son relevantes considerando la actualidad del problema abordado y la ausencia de investigaciones similares en la región. De frente al futuro, la innovación disruptiva debe priorizar el desarrollo humano, más allá de lo escolar, curricular e institucional, a fin de responder a las exigencias del porvenir. La pandemia reveló la urgencia de orientar los procesos formativos hacia nuevas formas de ser y estar en el mundo; de promover en la formación docente un giro pedagógico con énfasis en la justicia social.

Finalmente, la pandemia también representa una oportunidad para la generación de conocimiento sobre los aprendizajes construidos. Ahora se requiere recuperar, sistematizar y capitalizar la experiencia que demuestra la resiliencia y compromiso docentes frente a los momentos de dificultad. Del ejercicio se trazan nuevas líneas de investigación, relacionadas a la equidad e inclusión del profesorado con mayor vulnerabilidad, la atención al estudiantado con discapacidad, los resultados de las iniciativas implementadas, entre otros núcleos problemáticos.

De todo esto, estamos extrayendo una gran lección en el ámbito educativo. En este tiempo estamos reafirmando la idea de que la escuela no puede centrarse en el saber, sino en el hacer, el ser y el vivir juntos. La pandemia nos invita a replantear nuestro sistema educativo, redefinir las filosofías educativas que lo orientan, promover un nuevo humanismo, impulsar la equidad educativa, atender los vacíos en la formación pedagógica de los maestros y maestras 
en las escuelas normales. Como docente pienso que debemos aprender a educar en la era digital, pero no debemos olvidar que las TIC son medios y no fines, y que un reto adicional es superar la exclusión tecnológica aun prevaleciente en México, sin olvidar que el maestro es irremplazable y su labor una tarea fundamental. (Docente, ENYP 2h30m12s)

\section{Referencias}

Allen, C. (1996). What's wrong with the 'Golden Rule'? Conundrums of conducting ethical research in cyberspace. The Information Society, 12(2), 175-188. doi: https://doi.org/10.1080/713856146

Alves, A. I., Feraz, G. d., y Prudente, T. C. (2020). Formação humana em tempos de pandemia: os docentes, sua vida e trabalho. Revista Polyphonía, 31(1), 385-406. doi: https://doi.org/10.5216/ rp.v31i1.66968

Asociación Nacional de Universidades e Instituciones de Educación Superior (ANUIES). (2001). Programas Institucionales de Tutoría. Una propuesta de la ANUIES para su organización y funcionamiento en las instituciones de educación superior. ANUIES. https://bit.ly/3pxq1GN

Bardin, L. (2002). Análisis de contenido. Ediciones Akal.

Cervantes, E. (2020). Los aprendizajes no-académicos en la formación del profesorado de educación básica. Sinéctica. Revista electrónica de educación (55), 1-19. doi: https://doi.org/10.31391/ s2007-7033(2020)0055-011

Cervantes, E., y Sandoval, J. (2011). Procesos de formación docente en Chihuahua. Estado de conocimiento. XI Congreso Nacional de Investigación Educativa (pp. 1-9). México, D.F.: Consejo Mexicano de Investigación Educativa (COMIE). https://bit.ly/3k1GL7L

Chávez, P. G., Sáenz, A., y De León, J. E. (2017). La autoevaluación de competencias profesionales docentes de la Escuela Normal Rural "Ricardo Flores Magón" de Saucillo, Chih. 1er Congreso Nacional de Investigación sobre Educación Normal (pp. 1-12). SEP.

Comisión Nacional para la Mejora Continua de la Educación (MEJOREDU). (2020a). Indicadores nacionales de la mejora continua de la educación en México 2020. Cifras del ciclo escolar 2018-2019. MEJOREDU. https://bit.ly/3d9Llzw

Comisión Nacional para la Mejora Continua de la Educación (MEJOREDU). (2020b). 10 sugerencias para la educación durante la emergencia por el Covid-19. SEP. https://bit.ly/3702EiM

Comité para la Evaluación de Programas de Pedagogía y Educación (CEPPE). (2015). Marco de referencia para la acreditación de programas universitarios. CEPPE.

Cordero, G., Jiménez, J. A., Navarro, C., y Vázquez, M. d. (2017). Diagnóstico de la política pública de formación y desarrollo profesional del personal educativo de educación básica de la reforma educativa. INEE. https://bit.ly/2N7exg9

Corporación Andina de Fomento (CAF). (2020). Las oportunidades de la digitalización en América Latina frente al Covid-19. CAF. https://bit.ly/2NpccwO

Cortazar, S., y Aguirre, E. (2021). Una Mystory sobre la frustración de sentir la tesis como un proceso maquilado. Revista Argentina de Investigación Narrativa, 1(1), 250-265. https://bit.ly/2OwmqvH 
Cruz, K., y Delgado, C. (2019). La investigación y tutoría. Competencias del docente normalista. 3er Congreso Nacional de Investigación sobre Educación Normal (pp. 1-11). SEP. https://bit.ly/2NzHHnQ

Day, C., y Gu, Q. (2016). Educadores resilientes, escuelas resilientes. Construir y sostener la calidad educativa en tiempos difíciles. Narcea Ediciones.

Díaz-Barriga, F., y Barrón-Tirado, M. C. (2020). Currículo y pandemia: Tiempo de crisis y oportunidad de disrupción. Revista Electrónica Educare, 24(Suplemento), 1-5. doi: https://doi.org/10.15359/ ree.24-S.3

Estalella, A., y Ardèvol, E. (2007). Ética de campo: hacia una ética situada para la investigación etnográfica de internet. Forum: Qualitative Social Research, 8(3), Art. 2. https://bit.ly/3nr644R

Flores, V. J., García, I., y Romero, S. (2017). Prácticas inclusivas en la formación docente en México. Liberabit, 23(1), 39-56. doi: https://doi.org/10.24265/liberabit.2017.v23n1.03

Gibbs, G. (2012). El análisis de datos cualitativos en Investigación Cualitativa. Ediciones Morata.

González, O., González, M., y Ruiz, J. C. (2012). Consideraciones éticas en la investigación pedagógica: una aproximación necesaria. EDUMECENTRO, 4(1), 1-5. https://bit.ly/3tIoQqA

Instituto Nacional para la Evaluación de la Educación (INEE). (2018a). Directrices para mejorar las políticas de formación y desarrollo profesional docente en la educación básica. INEE.

Instituto Nacional para la Evaluación de la Educación (INEE). (2018b). Políticas para el mejoramiento profesional de los docentes en México. INEE. https://bit.ly/2ZjEr2C

Jurado, N., Jurado, O. I., y Sánchez, G. (2017). Las encrucijadas de la actualización en Educación Normal. 1er Congreso Nacional de Investigación sobre Educación Normal (pp. 1-12). SEP. https://bit. $1 y / 3 p E D 21 q$

López, I., Mella, J., y Cáceres, G. (2018). La universidad como ruptura en la trayectoria educativa: experiencias de transición de estudiantes egresados de Enseñanza Media Técnico Profesional que ingresan al Programa Académico de Bachillerato de la Universidad de Chile. Estudios Pedagógicos, 44 (3), 271-288. https://bit.ly/3mwKeM7

Murillo, F. J., y Duk, C. (2020). El Covid-19 y las Brechas Educativas. Revista Latinoamericana de Educación Inclusiva, 14(1), 11-13. doi: http://dx.doi.org/10.4067/S0718-73782020000100011

Navarro, M. A. (2020). Mejorar la formación docente. El heraldo de Chihuahua. https://bit.ly/3s9O682

Núñez, L. D., Burrola, L. M., y Morales, L. A. (2017). El perfil deseable de los maestros para la formación docente en las escuelas normales del estado de Chihuahua. 1er Congreso Nacional de Investigación sobre Educación Normal (pp. 1-14). SEP. https://bit.ly/3k0cdDo

Oficina Regional de Educación para América Latina y el Caribe de la Organización de las Naciones Unidas para la Educación, la Ciencia y la Cultura (OREALC/UNESCO Santiago). (2020). La educación en tiempos de la pandemia de COVID-19. OREALC/UNESCO. https://bit.ly/3pzyCd9

Organización de las Naciones Unidas para la Educación, la Ciencia y la Cultura (UNESCO). (2019). Marco de competencias de los docentes en materia de TIC. UNESCO. https://bit.ly/3rZ6YH3

Ortega, F. J. (2020). Participación individual. Encuentro virtual de instituciones de posgrado. El trabajo docente no presencial en el periodo de contingencia sanitaria por el SARS-COV2 [Video]. SEyD. https://bit.ly/37

Ruiz, A. (2006). Texto, testimonio y metatexto. El análisis de contenido en la investigación en educación. En A. Jiménez, y A. Torres, La práctica investigativa en ciencias sociales (pp. 45-62). Universidad Pedagógica Nacional (UPN). https://bit.ly/3k9OwsA 
Santibáñez, L., Rubio, D., y Vázquez, M. (2018). Formación Continua de Docentes: Política actual en México y buenas prácticas nacionales e internacionales. INEE. https://bit.ly/2NkYGdF

Secretaría de Educación Pública (SEP). (2017a). Modelo educativo para la educación obligatoria. Educar para la libertad y la creatividad. SEP. https://bit.ly/3dtkL2D

Secretaría de Educación Pública (SEP). (2017b). Aprendizajes clave para la educación integral. Plan y programas de estudio para la educación básica. SEP. https://bit.ly/3qvZ8o2

Secretaría de Educación y Deporte (SEyD). (2020a). Encuentro Virtual de Instituciones Formadoras y Actualizadoras de Docentes [Video]. YouTube: https://bit.ly/2LsdYvT

Secretaría de Educación y Deporte (SEyD). (2020b). Encuentro virtual de instituciones de posgrado. El trabajo docente no presencial en el periodo de contingencia sanitaria por el SARS-COV2 [Video]. YouTube: https://bit.ly/37fguhO

Secretaría de Educación y Deporte (SEyD). (12 de noviembre de 2020c). Realizarán Encuentro Virtual de Instituciones Formadoras y Actualizadoras de Docentes. Cambio. https://bit.ly/2R1mWDd

Tójar, J. C. (2006). Investigación cualitativa: comprender y actuar. La Muralla.

Trujillo, J. A., Pérez, F. A., y Hernández, G. (2018). La formación de maestros en Chihuahua, México: una disputa por el proyecto ideológico de Estado a través de la escuela. XIII Congreso Iberoamericano de Historia de la Educación Latinoamericana (pp. 1-2). Sociedad Uruguaya de Historia de la Educación (SUHE). https://bit.ly/2NBuif8

Vezub, L. (2020). Argentina. Formación docente situada [Video]. MEJOREDU. YouTube https://bit. ly/2UzvOhO 